\title{
Percoll-Purified Treponema pallidum, an Improved Fluorescent Treponemal Antibody-Absorbed Antigen
}

\author{
PHILIP A. HANFF,${ }^{1}+*$ CONCHITA FERNANDEZ, ${ }^{1} \ddagger$ AND JAMES D. FOLDS ${ }^{1,2}$ \\ Clinical Microbiology and Immunology Laboratories, Division of Laboratory Medicine, North Carolina Memorial \\ Hospital, ${ }^{1}$ and Department of Microbiology and Immunology, University of North Carolina, ${ }^{2}$ Chapel Hill,
} North Carolina 27514

Received 19 September 1985/Accepted 13 January 1986

\begin{abstract}
Percoll-purified Treponema pallidum was evaluated as a fluorescent treponemal antibody-absorbed antigen. Borderline and false-positive reactions were essentially eliminated, resulting in sensitivity and specificity of 100 and $95.5 \%$, respectively. The lack of background debris improved the ease and speed of reading the test.
\end{abstract}

Since the introduction of the fluorescent treponemal antibody (FTA) test for the serodiagnosis of syphilis (2), the preparation of Treponema pallidum antigen from infected rabbit testicular tissue has not substantially changed. This is despite the development of the hemagglutination $(15,17,18$, $22)$ and enzyme immunoassay $(10,14,20)$ tests and modifications to the FTA test $(7,9,11)$. The lack of change has been due, in part, to the difficulties associated with obtaining large quantities of purified antigen that are free of contaminating host testicular tissue and retain morphological characteristics and immunological reactivity.

Although Hunter et al. (7) standardized methodology for preparing FTA-absorbed (ABS) antigen that was free of rabbit globulin, sensitive techniques revealed that these $T$. pallidum preparations were still contaminated with rabbit testicular material (5). The development of the continuous particle electrophoresis method for separation of $T$. pallidum from testicular material (16) resulted in the availability of a purified antigen for use in the FTA-ABS test. This continuous particle electrophoresis antigen is no longer available, and its preparation was technically difficult and expensive. Furthermore, it was a low-yield procedure that resulted in nonmotile, avirulent, and ultrastructurally damaged organisms $(5,16)$. The possibility exists, therefore, that antigenic changes accompanying cell death were responsible for lotto-lot variability in FTA-ABS reactivity.

A new, rapid purification technique utilizing Percoll density gradient centrifugation has been described recently (5). The organisms were shown to be ultrastructurally intact, motile, virulent, and free of detectable soluble and insoluble host testicular material. In this report we evaluate the use of Percoll-purified $T$. pallidum as an FTA-ABS antigen. The evidence presented suggests that the purity and quality of the $T$. pallidum antigen preparation may have a direct effect on the occurrence of equivocal and borderline reactions.

T. pallidum, Nichols strain, was maintained without corticosteroids and harvested in FTA-ABS buffer (CSI, Inc., Whippany, N.J.) as previously described (5). To increase bacterial yields for preparation of FTA-ABS antigen, rabbits were given cortisone acetate from days 3 to 10 postinfection (19). Soluble and insoluble host testicular tissue was re-

\footnotetext{
* Corresponding author.

† Present address: Clinical Microbiology, Beth Israel Hospital, Boston, MA 02215.

$\ddagger$ Present address: Cutter Biological, P. O. Box 1986, Berkeley, CA 94701 .
}

moved from treponemal suspensions by low-speed differential centrifugation followed by density gradient centrifugation through Percoll (Pharmacia Fine Chemicals, Piscataway, N.J.) as described elsewhere (5). Percoll was removed from the purified treponemes by centrifugation at $100,000 \times g$ for $1 \mathrm{~h}$ at $4^{\circ} \mathrm{C}(5)$, and the organisms were diluted $1: 20$ in lyophilization buffer $(8)$ and maintained at $4^{\circ} \mathrm{C}$ for 10 days. Samples $(0.5 \mathrm{ml})$ were lyophilized and stored at $4^{\circ} \mathrm{C}$ until used (8). Purified T. pallidum antigen was reconstituted in $0.5 \mathrm{ml}$ of double-distilled water and adjusted to a working concentration in FTA-ABS buffer. Before use, 10-well slides (Carlson Scientific, Peotone, Ill.) were washed in $7 \times$ detergent, rinsed in water, soaked in $95 \%$ ethanol, and allowed to air dry. Ten microliters of the treponemal suspension was placed in each well and allowed to air dry in a laminar-flow hood. Antigen-coated slides were fixed in acetone for $10 \mathrm{~min}$ and stored at $-20^{\circ} \mathrm{C}(21)$. Antigen testing and titration of the fluorescein-conjugated anti-human globulin (BBL Microbiology Systems, Cockeysville, Md.) were performed as described (21). The reactive control serum and a total of 122 diagnostically and serologically well-characterized sera from patients with syphilis and from patients with false-positive or nonspecific fluoresence in the FTA-ABS test were generously provided by Sandra Larsen (Treponemal Research Branch, Sexually Transmitted Disease Laboratory Program, Center for Infectious Disease, Centers for Disease Control $[\mathrm{CDC}]$ ). The sera were selected for use on the basis of their FTA-ABS reactivity. Approximately 20 sera each were categorized as nonreactive, borderline, or 1-, 2-, 3-, and 4-plus reactive. The FTA-ABS test was performed as described (21) and interpreted in a double-blind fashion by two experienced readers. A positive test was considered to be a result of 1 to $4+$. A typical $3+$ reaction is shown in Fig. 1 . Borderline reactions were noted but interpreted as negative (1).

The FTA-ABS test with the purified antigen (FTA-P) and the test performed at the CDC (FTA-CDC) detected 77 true positives (Table 1). False-positives occurred with both tests, but it is important to note that the false-positive results were obtained with different sera. It also appears that the ability of each test to correctly identify true negatives (normals and individuals with diseases other than syphilis) was similar.

A significant difference in the number of borderline reactions was observed, however. A total of 21 borderline reactions occurred with the FTA-CDC test (Table 1). This included 3 patients with treated latent syphilis, 1 with congenital syphilis, 3 false-positives, and 14 normal individ- 
TABLE 1. FTA-ABS testing with Percoll-purified $T$. pallidum

\begin{tabular}{|c|c|c|c|c|c|c|}
\hline \multirow{3}{*}{$\begin{array}{l}\text { Diagnostic } \\
\text { category }\end{array}$} & \multicolumn{6}{|c|}{ No. of sera by test ${ }^{a}$ : } \\
\hline & \multicolumn{3}{|c|}{ FTA-CDC } & \multicolumn{3}{|c|}{ FTA-P } \\
\hline & Reactive $^{b}$ & Borderline & Nonreactive & Reactive $^{b}$ & Borderline & Nonreactive \\
\hline Syphilis $^{c}$ & 77 & 4 & 0 & 77 & 1 & 3 \\
\hline Other diseases & 0 & 0 & 20 & 0 & 0 & 20 \\
\hline Normal & 0 & 14 & 0 & 2 & 1 & 11 \\
\hline False-positive & 4 & 3 & 0 & 0 & 0 & 7 \\
\hline
\end{tabular}

${ }^{a}$ FTA-ABS testing performed at North Carolina Memorial Hospital with Percoll-purified $T$, pallidum (FTA-P) or by CDC with nonpurified antigen (FTA-CDC).

${ }^{b}$ Reactive, $>1+$ reaction.

c The diagnosis of syphilis was based on detailed historical, clinical, and serologic information provided to or obtained by the CDC. There were 21 patients with untreated and treated early syphilis, 52 with untreated and treated latent disease, 7 with congenital syphilis, and 1 with late cardiovascular syphilis.

uals. When these sera were tested by the FTA-P 17 were nonreactive, 2 remained borderline, and 2 became reactive. Similarly, seven sera that were diagnostically categorized as false-positives were nonreactive in the FTA-P. In contrast, one $2+$, three $1+$, and three borderline reactions occurred with the FTA-CDC. The sensitivity and specificity of the FTA-P were 100 and $95.5 \%$, and those of the FTA-CDC were 98.7 and $90.9 \%$, respectively.

All treponemal tests for syphilis utilize treponemal antigens that are contaminated with rabbit testicular material. The inability to obtain sufficient quantities of purified antigen results from the fact that only limited in vitro multiplication of the organism has been reported $(3,4,12,13)$. Furthermore, an efficient method for purification of $T$. pallidum from host tissue has not been available. Recently, however, the separation of soluble and insoluble host material from $T$. pallidum suspensions by Percoll density gradient centrifugation was described (5). The resultant organisms were biologically, morphologically, and immunologically intact. Preliminary evidence suggested that the use of Percoll-purified $T$. pallidum as an FTA-ABS antigen reduced the number of

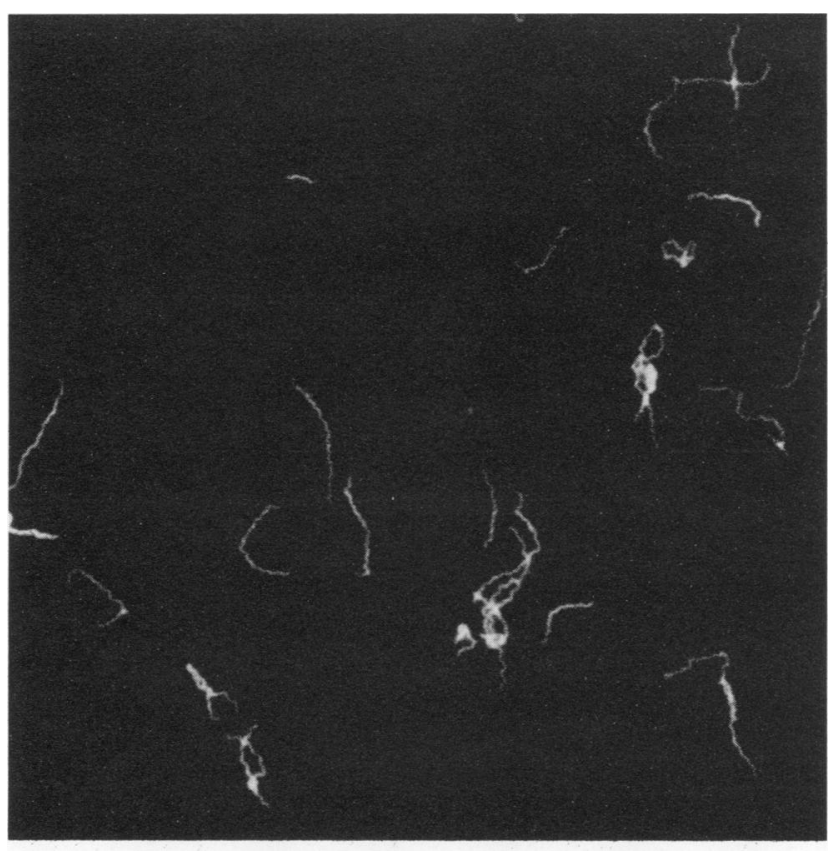

FIG. 1. Percoll-purified T. pallidum as an FTA-ABS antigen. equivocal reactions and enhanced the ease and speed of reading (P. A. Hanff, C. Fernandez, J. Waters, and J. D. Folds, Progr. Abstr. 83rd Intersci. Conf. Antimicrob. Agents Chemother., abstr. no. 669, 1983). This appeared to be due, in part, to the purity of the antigen and the resultant lack of background debris (5). Additionally, Percoll-purified antigen remained firmly fixed to the slides throughout the procedure. In contrast, some loss of the standard nonpurified antigen is expected $(6,21)$.

In an attempt to demonstrate more accurately that Percollpurified $T$. pallidum could be effectively utilized as an FTA-ABS antigen, we tested diagnostically and serologically well-characterized sera, using lyophilized purified antigen. The sensitivity and specificity of the FTA-P and the FTA-CDC were essentially similar: 100 and $95.5 \%$ versus 98.7 and $90.9 \%$, respectively. There was a marked difference, however, in the number of borderline reactions observed with the two tests. Of the 21 borderline results measured by the CDC, $19(95 \%)$ were nonreactive by the FTA-P, and 2 became positive. The CDC currently recommends that borderline reactions be interpreted as negative (1). Thus, the differences observed between the FTA-P and FTA-CDC may not be clinically significant.

In contrast, the fact that all patients classified as falsepositive were nonreactive with the FTA-P may have important clinical and serologic implications. We have shown that the quality and purity of the $T$. pallidum antigen have an effect on borderline and false-positive FTA-ABS results. Similar results have been obtained by comparing the reactivity of a commercial FTA-ABS antigen with nonpurified and Percoll-purified $T$. pallidum prepared from the same suspension (G. Tringali, P. A. Hanff, G. Scuderi, and G. Fragale, submitted for publication). If these results are confirmed in larger prospective evaluations utilizing FTAABS, hemagglutination, or enzyme-linked immunosorbent assays, it is conceivable that many of the interpretive problems encountered with the treponemal tests for syphilis and the resultant diagnostic dilemmas will be resolved.

We thank Elizabeth Hunter for invaluable conversations and guidance, Jennifer Waters for technical assistance, and Lydia Moretti for excellent secretarial assistance.

\section{LITERATURE CITED}

1. Centers for Disease Control, Biological Products Program. 1984. Current status of FTA-ABS tests for syphilis. Supplement no. 27. Centers for Disease Control, Atlanta, $\mathrm{Ga}$.

2. Deacon, W. E., V. H. Falcone, and A. Harris. 1957. A fluorescent test for treponemal antibodies. Proc. Soc. Exp. Biol. Med. 96:477-490. 
3. Fieldsteel, A. H., D. L. Cox, and R. A. Moeckli. 1981. Cultivation of virulent Treponema pallidum in tissue culture. Infect. Immun. 32:908-915.

4. Fieldsteel, A. H., D. L. Cox, and R. A. Moeckli. 1982. Further studies on replication of virulent Treponema pallidum in tissue cultures of Sf1EP cells. Infect. Immun. 35:449-455.

5. Hanff, P. A., S. J. Norris, M. A. Lovett, and J. N. Miller. 1984. Purification of Treponema pallidum, Nichols strain, by Percoll density gradient centrifugation. Sex. Transm. Dis. 11:275-286.

6. Hunter, E. F., M. R. Adams, L. H. Orrison, B. J. Pender, and S. A. Larsen. 1979. Problems affecting performance of the fluorescent treponemal antibody-absorption test for syphilis. J. Clin. Microbiol. 9:163-169.

7. Hunter, E. F., E. T. Creighton, and J. S. Lewis. 1970. An improved antigen for the FTA-ABS test. Health Lab. Sci. 7:237-241.

8. Hunter, E. F., E. T. Creighton, and J. S. Lewis. 1971. A lyophilization medium for FTA-ABS test antigen. Health Lab. Sci. 8:35-39.

9. Hunter, E. F., W. E. Deacon, and P. E. Meyer. 1964. An improved FTA test for syphilis, the absorption procedure (FTAABS). Public Health Rep. 79:410-412.

10. Hunter, E. F., C. E. Farshy, S. L. Liska, D. D. Cruce, J. A. Crawford, and J. C. Feeley. 1982. Sodium desoxycholateextracted treponemal antigen in an enzyme-linked immunosorbent assay for syphilis. J. Clin. Microbiol. 16:483-486.

11. Hunter, E. F., R. M. McKinney, S. E. Maddison, and D. D. Cruce. 1979. Double-staining procedure for the fluorescent treponemal antibody absorption (FTA-ABS) test. Br. J. Vener. Dis. 55:105-108.

12. Levy, J. A. 1984. Confirmation of the successful cultivation of Treponema pallidum in tissue culture. Microbiologica 7:367-370.
13. Norris, S. J. 1982. In vitro cultivation of Treponema pallidum: independent confirmation. Infect. Immun. 36:437-439.

14. Pope, V., E. F. Hunter, and J. C. Feeley. 1982. Evaluation of the microenzyme-linked immunosorbent assay with Treponema pallidum antigen. J. Clin. Microbiol. 15:630-634.

15. Rathlev, T. 1967. Hemagglutination test utilizing pathogenic Treponema pallidum for the sero-diagnosis of syphilis. Br. J. Vener. Dis. 43:181-185.

16. Schmale, J. D., D. S. Kellogg, Jr., C. E. Miller, P. Schammel, and J. D. Thayer. 1970. Separation of Treponema pallidum from tissue debris through continuous-particle electrophoresis. Appl. Microbiol. 19:287-289.

17. Tomizawa, T., and S. Kasamatsu. 1966. Hemagglutination tests for the diagnosis of syphilis. A preliminary report. Jpn. J. Med. Sci. Biol. 19:305-308.

18. Tomizawa, T., S. Kasamatsu, and S. Yamaya. 1969. Usefulness of the hemagglutination test using Treponema pallidum antigen (TPHA) for the serodiagnosis of syphilis. Jpn. J. Med. Sci. Biol. 22:341-350.

19. Turner, T. B., and D. H. Hollander. 1954. Studies on the mechanism of action of cortisone in experimental syphilis. $J$. Syph. Gonorrhea Vener. Dis. 38:371-387.

20. Veldkamp, J., and A. M. Visser. 1975. Application of the enzyme-linked immunosorbent assay (ELISA) in the serodiagnosis of syphilis. Br. J. Vener. Dis. 51:227-231.

21. Venereal Disease Program, National Communicable Disease Center. 1969. Manual of tests for syphilis. U. S. Government Printing Office, Washington, D.C.

22. Wentworth, B. B., M. A. Thompson, C. R. Peter, R. E. Bawson, and D. L. Wilson. 1978. Comparison of a hemagglutination treponemal test for syphilis (HATTS) with other serologic methods for the diagnosis of syphilis. Sex. Transm. Dis. 5:103-111. 